\title{
Influence of Thermal Radiation on Unsteady Free Convection MHD Flow of Brinkman Type Fluid in a Porous Medium with Newtonian Heating
}

\author{
Farhad Ali, ${ }^{1}$ Ilyas Khan, ${ }^{1,2}$ Sami U1 Haq, ${ }^{1}$ and Sharidan Shafie ${ }^{1}$ \\ ${ }^{1}$ Department of Mathematical Sciences, Faculty of Science, Universiti Teknologi Malaysia (UTM), 81310 Johor Bahru, Johor, Malaysia \\ ${ }^{2}$ College of Engineering Majmaah University, P.O. Box 66, Majmaah 11952, Saudi Arabia \\ Correspondence should be addressed to Sharidan Shafie; sharidan@utm.my
}

Received 20 June 2013; Revised 13 September 2013; Accepted 13 September 2013

Academic Editor: Waqar Khan

Copyright (C) 2013 Farhad Ali et al. This is an open access article distributed under the Creative Commons Attribution License, which permits unrestricted use, distribution, and reproduction in any medium, provided the original work is properly cited.

\begin{abstract}
The focus of this paper is to analyze the influence of thermal radiation on some unsteady magnetohydrodynamic (MHD) free convection flows of an incompressible Brinkman type fluid past a vertical flat plate embedded in a porous medium with the Newtonian heating boundary condition. The fluid is considered as a gray absorbing-emitting but nonscattering medium and the Rosseland approximation in the energy equations is used to describe the radiative heat flux for optically thick fluid. For a detailed analysis of the problem, four important situations of flow due to (i) impulsive motion of the plate (ii) uniform acceleration of the plate (iii) nonuniform acceleration of the plate, and (iv) highly nonuniform acceleration of the plate are considered. The governing equations are first transformed into a system of dimensionless equations and then solved analytically using the Laplace transform technique. Numerical results for temperature and velocity are shown graphically, while skin friction and Nusselt number are computed in tables. The results show that temperature and velocity increase on increasing radiation and Newtonian heating parameters. However, the results of magnetic and porosity parameters on velocity are found quite opposite.
\end{abstract}

\section{Introduction}

Generally speaking, a free convection flow field is a selfsustained flow driven by the presence of a temperature gradient. Extensive research work has been published on free convection flow past a vertical plate for different boundary conditions (see [1-10] and the references therein). Perhaps, it is due to their numerous applications in engineering, geophysical and astrophysical environments, geothermal energy, oil reservoir modelling, building insulation, food processing, and grain storage. Most of these existing studies have used the usual boundary conditions of constant wall temperature or constant heat flux. However, in many practical situations it was realized that the convection flow can also be set up by the Newtonian heating from the surface known as conjugate convective flow [11]. The Newtonian heating situation occurs in many important engineering devices such as in heat exchanger where the conduction in solid tube wall is greatly influenced by the convection in the fluid flowing over it and in conjugate heat transfer around fins where the conduction within the fin and the convection in the fluid surrounding it are simultaneously analyzed in order to obtain the vital design information [12]. In view of these applications various researchers are involved in dealing with the Newtonian heating problems via numerical or analytical techniques.

Chaudhary and Jain $[13,14]$ investigated the problem of unsteady free convection flow of an incompressible viscous fluid for different motions of the plate with the Newtonian heating condition and obtained the exact solutions using the Laplace transform technique. Recently, Guchhait et al. [15] reinvestigated the problem of Chaudhary and Jain [14] numerically by using the Crank-Nicolson implicit finitedifference scheme. Mebine and Adigio [16] obtained an exact solution for unsteady free convection flow of an incompressible viscous optically thin fluid past an impulsively started vertical porous plate with the Newtonian heating. Salleh et al. 
$[17,18]$ analyzed numerically the steady boundary layer flow and heat transfer over a horizontal circular cylinder and over a stretching sheet with the Newtonian heating. Narahari and Ishak [19] investigated the influence of thermal radiation on unsteady free convection flow past a moving vertical plate with Newtonian heating. Recently Akbar and Nadeem [20] studied mixed convective MHD peristaltic flow of a Jeffrey nanofluid with the Newtonian heating. Narahari and Yunus [21] performed an analytical study of free convection flow near an impulsively started infinite vertical plate with Newtonian heating in the presence of thermal radiation and constant mass diffusion. The closed-form exact solutions were obtained by the Laplace transform method.

On the other hand, the study of MHD flows has important technological and geothermal applications in cooling of nuclear reactors, liquid metals fluid, power generation system, aerodynamics, and engineering problems. The study of MHD flow with heat transfer also plays an important role in biological sciences. In addition, the flow through a porous medium is of great physical interest because of its various applications found in hydrology for the sand structures in earth, in filter beds for purification of drinking water and sewage, in chemical engineering for the permeation chromatography, filtering of gases, and liquids chromatography, and in petroleum technology for the production of petroleum and gases. Having such motivation, Hayat et al. [22] analyzed MHD stagnation point flow of a Jeffery fluid past a stretching surface with the Newtonian heating. Lesnic et al. [23, 24] analyzed the free convection flow in a porous medium generated by Newtonian heating. In another study, Lesnic et al. [25] considered the steady free convection boundary layer flow along a semi-infinite plate, slightly inclined to the horizontal and embedded in a porous medium with the flow generated by Newtonian heating. Furthermore, radiative convective flows are frequently encountered in many environmental and scientific processes such as aeronautics, fire research, and heating and cooling of channels. Radiation and thermal diffusion effects on MHD free convection flow of an incompressible viscous fluid near an oscillating plate embedded in a porous medium were considered by Khan et al. [26]. Radiation and porosity effects on the MHD flow past an oscillating vertical plate with uniform heat flux were presented by Samiulhaq et al. [27]. Mohammed et al. [28] discussed analytical solutions of MHD natural convection transients flow near an oscillating plate emerged in a porous medium.

Most of the above studies with Newtonian heating conditions are performed for viscous fluids and numerical or exact solutions are obtained. However, such studies for nonNewtonian fluids are very few, especially those that are solved for exact solutions. Non-Newtonian fluids problems on the other hand are of great and increasing interest for the last five decades. Such fluids differ from the Newtonian fluids in that the relationship between the shear stress and flow field is more complicated. Examples of the non-Newtonian fluids are coal water, food products, inks, glues, soaps, and polymer solutions [29-33]. Therefore, in the present investigation we have chosen Brinkman type fluid as a non-Newtonian fluid. This model was first used by Brinkman in [34, 35]. Later, several authors have used the Brinkman model in describing several types of flows through a porous medium. Among them, Hsu and Cheng [36] studied the natural convection about a semi-infinite vertical flat plate in a porous medium using Brinkman model. Varma and Babu [37], Gorla et al. [38], and Rajagopal [39] also used Brinkman model in their studies. By using Brinkman type fluid, Fetecǎu et al. [40] reported some interesting results. They used Fourier sine transform and obtained exact solutions for some unsteady motions. Recently, this problem was reinvestigated by Ali et al. [41] and they established some new types of exact solutions using Laplace transform.

Motivated by above studies, the present research aims to analyze the influence of thermal radiation on unsteady MHD free convection flow of Brinkman type fluid past a vertical plate embedded in a porous medium with Newtonian heating. To the best of authors' knowledge such study is not available in the literature and the present work claims to fill this space. Moreover, for a detailed analysis of the problem four different types of plate motion are considered. As a result, the following flow situations known as (i) flow induced by an impulsive motion of the plate, (ii) flow due to uniform acceleration of the plate, (iii) flow due to nonuniform acceleration of the plate, and (iv) flow due to highly nonuniform acceleration of the plate are discussed. Exact solutions of momentum and energy equations are obtained by using Laplace transform technique. They satisfy all imposed initial and boundary conditions and can easily be reduced to the similar solutions for hydrodynamic Brinkman type and Newtonian fluids. Analytical as well as numerical results for skin friction and Nusselt number are provided. Graphical results are presented and discussed for various physical parameters. Applications of the present problem are diverse. Because this work presents a new research on free convection flow of Brinkman type fluid for some fundamental fluid motions, therefore this problem can be extended for more complicated studies on Brinkman type fluid in various porous media. Furthermore, the exact solutions obtained in this study will assist scientist and engineers as they can use them as a check of correctness for numerical or approximate solutions of complicated flow problems.

\section{Mathematical Formulation and Solution of the Problem}

Let us consider unsteady free convection flow of an incompressible and electrically conducting fluid of Brinkman type [40]. It is assumed that the plate occupies the half porous space $y>0$ over an infinite vertical flat plate situated in the $(x, z)$ plane of a Cartesian coordinate system $x, y$, and $z$. The $x$-axis is taken along the vertical plate and the $y$ axis is taken normal to the plate. A uniform magnetic field of strength $B_{0}$ is transversely applied to the plate in the outward direction. The applied magnetic field is assumed to be strong enough so that the induced magnetic field due to the fluid motion is weak and can be neglected. As mentioned by Cramer and Pai [42], this assumption is physically justified for partially ionized fluids and metallic 


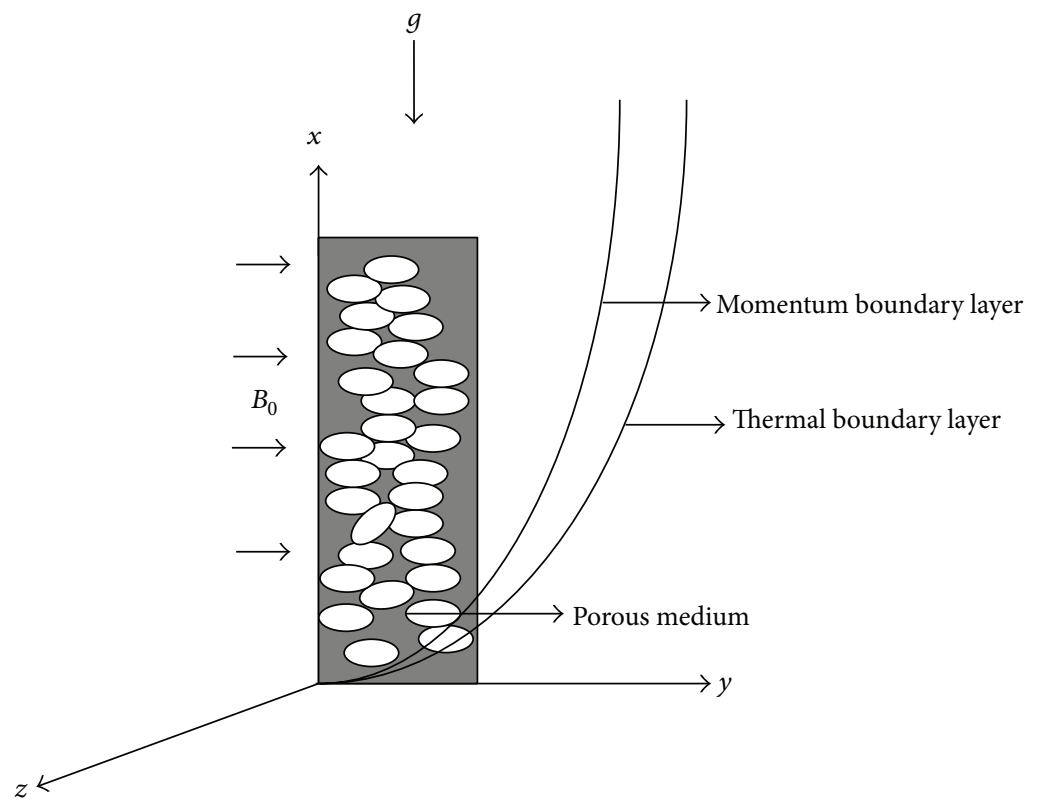

Figure 1: Physical model and coordinates system.

liquids because of their small magnetic Reynolds number. Since there is no applied or polarization voltage imposed on the flow field, therefore the electric field due to polarization of charges is zero. Initially, both the plate and fluid are at rest with constant temperature $T_{\infty}$. After time $t=0^{+}$, the flow is caused by translating the plate in its plane with timedependent velocity $A t^{n}$ against the gravitational field. It is assumed that the rate of heat transfer from the surface is directly proportional to the local surface temperature $T$. Due to the shear, the fluid is gradually moved and its velocity is of the form

$$
\mathbf{v}=\mathbf{v}(y, t)=u(y, t) \mathbf{i}
$$

where $\mathbf{i}$ is the unit vector in the flow direction. The physical model is shown in Figure 1.

In addition to the above assumptions, we assume that the internal dissipation is absent and the usual Boussinesq approximation is taken into consideration. Moreover, the pressure gradient in the flow direction is compensated by the gradient of the hydrostatic pressure gradient of the fluid. As a result the governing equations of momentum and energy are derived as follows:

$$
\begin{aligned}
\frac{\partial u(y, t)}{\partial t}+\beta u(y, t)= & \nu \frac{\partial^{2} u(y, t)}{\partial y^{2}}-\frac{\sigma B_{0}^{2} u(y, t)}{\rho} \\
& -\frac{\nu \phi u(y, t)}{k}+g \beta_{T}\left(T-T_{\infty}\right), \\
\rho c_{p} \frac{\partial T}{\partial t} & =k_{1} \frac{\partial^{2} T}{\partial y^{2}}-\frac{\partial q_{r}}{\partial y}
\end{aligned}
$$

where $T$ is the temperature of the fluid, $\nu=\mu / \rho$ ( $\mu$ is the viscosity and $\rho$ is the constant density of the fluid) is the kinematic viscosity, $\sigma$ is the electrical conductivity of the fluid, $\beta=\alpha / \rho$ ( $\alpha$ is the $\operatorname{drag}$ coefficient that is usually assumed to be positive), $\phi$ is the porosity parameter, $k$ is the permeability parameter, $\beta_{T}$ is the volumetric coefficient of thermal expansion, $g$ is the acceleration due to gravity, $T_{\infty}$ is the free stream temperature, $c_{p}$ is the specific heat of the fluid at a constant pressure, $k_{1}$ is the thermal conductivity, and $q_{r}$ is the radiative flux along the $y$-axis.

The initial and boundary conditions relevant to the present flow situation are

$$
\begin{gathered}
t \leq 0: u(y, t)=0, \quad T(y, t)=T_{\infty} ; \quad y>0, \\
t>0: u(0, t)=A t^{n}, \quad \frac{\partial T(0, t)}{\partial y}=-h_{s} T, \\
u(\infty, t)=0, \quad T(\infty, t)=T_{\infty},
\end{gathered}
$$

where $n \geq 0$ is constant and $h_{s}$ is the heat transfer parameter for Newtonian heating. The radiative heat flux term in (3) is simplified using the Rosseland approximation as

$$
q_{r}=-\frac{4 \sigma_{1}}{3 k_{2}} \frac{\partial T^{4}}{\partial y},
$$

where $\sigma_{1}$ is the Stefan-Boltzmann constant and $k_{2}$ is the mean absorption coefficient. It is assumed that the temperature differences inside the flow are sufficiently small such that $T^{4}$ may be expressed as a linear function of the temperature, and then by expanding $T^{4}$ about $T_{\infty}$ using Taylor series and neglecting the higher order terms, we get

$$
T^{4} \cong 4 T_{\infty}^{3} T-3 T_{\infty}^{4} .
$$

Now (3) after using (5) and (6) takes the following form:

$$
\rho c_{p} \frac{\partial T}{\partial t}=k_{1}\left(1+\frac{16 \sigma T_{\infty}^{3}}{3 k_{1} k_{2}}\right) \frac{\partial^{2} T}{\partial y^{2}} .
$$


By introducing the following dimensionless variables

$$
\begin{gathered}
v_{n}=\frac{u}{\left(\nu^{n} A\right)^{1 /(2 n+1)}}, \quad \xi=y\left(\frac{A}{v^{n+1}}\right)^{1 /(2 n+1)}, \\
\tau=t\left(\frac{A^{2}}{v}\right)^{1 /(2 n+1)}, \\
\beta_{n}=\beta\left(\frac{v}{A^{2}}\right)^{1 /(2 n+1)}, \quad \theta=\frac{T-T_{\infty}}{T_{\infty}},
\end{gathered}
$$

into (2)-(4) and (7), we obtain the following dimensionless system:

$$
\begin{gathered}
\frac{\partial v_{n}(\xi, \tau)}{\partial \tau}=\frac{\partial^{2} v_{n}(\xi, \tau)}{\partial \xi^{2}}-\beta_{n} v_{v}(\xi, \tau) \\
-H v_{n}(\xi, \tau)+\operatorname{Gr} \theta(\xi, \tau), \\
\operatorname{Pr} \frac{\partial \theta}{\partial \tau}=(1+R) \frac{\partial^{2} \theta}{\partial \xi^{2}}, \\
\tau \leq 0: v_{n}(\xi, \tau)=0, \quad \theta(\xi, \tau)=0 ; \quad \xi>0, \\
\tau>0: v_{n}(0, \tau)=\tau^{n}, \quad \frac{\partial \theta(0, \tau)}{\partial \xi}=-\gamma(1+\theta), \\
v_{n}(\infty, \tau)=0, \quad \theta(\infty, \tau)=0,
\end{gathered}
$$

where $\gamma$ is the Newtonian heating parameter. Using the Laplace transform technique, (9)-(11) give the following transformed solutions:

$$
\begin{aligned}
\bar{v}_{n}(\xi, q)= & \frac{\Gamma(n+1)}{q^{n+1}} e^{-\xi \sqrt{q+H}}+a_{1} \frac{e^{-\xi \sqrt{q+H}}}{\sqrt{q}-d}+a_{2} \frac{e^{-\xi \sqrt{q+H}}}{q} \\
& +a_{3} \frac{e^{-\xi \sqrt{q+H}}}{\sqrt{q}}-a_{4} \frac{e^{-\xi \sqrt{q+H}}}{q-H_{1}}-a_{5} \frac{\sqrt{q} e^{-\xi \sqrt{q+H}}}{q-H_{1}} \\
& -a_{1} \frac{e^{-\xi \sqrt{q a}}}{\sqrt{q}-d}-a_{2} \frac{e^{-\xi \sqrt{q a}}}{q}-a_{3} \frac{e^{-\xi \sqrt{q a}}}{\sqrt{q}} \\
& +a_{4} \frac{e^{-\xi \sqrt{q a}}}{q-H_{1}}+a_{5} \frac{\sqrt{q} e^{-\xi \sqrt{q a}}}{q-H_{1}} \\
& \bar{\theta}(\xi, q)=\frac{d}{q(\sqrt{q}-d)} e^{-\xi \sqrt{q a}}
\end{aligned}
$$

where

$$
\begin{gathered}
\beta_{n}=\beta\left(\frac{v}{A^{2}}\right)^{1 /(2 n+1)}, \quad M^{2}=\frac{\sigma B_{0}^{2}}{\rho}\left(\frac{v}{A^{2}}\right)^{1 /(2 n+1)}, \\
\frac{1}{K}=\frac{\nu \phi}{k}\left(\frac{v}{A^{2}}\right)^{1 /(2 n+1)}, \quad \mathrm{Gr}=\frac{g \beta_{T} T_{\infty}}{\left(A^{3} \nu^{n-1}\right)^{1 /(2 n+1)}}, \\
\gamma=h_{s}\left(\frac{\nu^{n+1}}{A}\right)^{1 /(2 n+1)}, \quad \operatorname{Pr}=\frac{\mu c_{p}}{k_{1}}, \quad R=\frac{16 \sigma T_{\infty}^{3}}{3 k_{1} k_{2}}, \\
H=M^{2}+\frac{1}{K}, \quad H_{1}=\frac{H}{a-1}, \\
\mathrm{Gr}_{1}=\frac{\mathrm{Gr}^{2}}{a-1}, \quad \mathrm{Pr}_{1}=\frac{\gamma}{\sqrt{a}}, \quad \frac{\mathrm{Gr}_{1}}{d^{2}\left(d^{2}-H_{1}\right)}, \\
a_{2}=\frac{\mathrm{Gr}_{1}}{d H_{1}}, \quad a_{3}=\frac{\mathrm{Gr}_{1}}{d^{2} H_{1}}, \\
a_{4}=\frac{\mathrm{Gr}_{1} d}{H_{1}\left(d^{2}-H_{1}\right)}, \quad a_{5}=\frac{\mathrm{Gr}_{1}}{H_{1}\left(d^{2}-H_{1}\right)} .
\end{gathered}
$$

The Laplace inverse of (13) results in

$$
\begin{aligned}
\theta(\xi, \tau)= & \exp \left(d^{2} \tau-y \sqrt{a} d\right) \operatorname{erf} c\left[\frac{y \sqrt{a}}{2 \sqrt{\tau}}-d \sqrt{\tau}\right] \\
& -\operatorname{erf} c\left[\frac{y \sqrt{a}}{2 \sqrt{\tau}}\right] .
\end{aligned}
$$

For a detailed analysis of (12), we are considering the following four cases.

\section{Flow Induced by an Impulsive Motion of the Plate}

For an impulsive motion of the plate $(n=0),(12)$ yields

$$
\begin{aligned}
\bar{v}_{0}(\xi, q)= & \frac{1}{q} e^{-\xi \sqrt{q+H}}+a_{1} \frac{e^{-\xi \sqrt{q+H}}}{\sqrt{q}-d}+a_{2} \frac{e^{-\xi \sqrt{q+H}}}{q} \\
& +a_{3} \frac{e^{-\xi \sqrt{q+H}}}{\sqrt{q}}-a_{4} \frac{e^{-\xi \sqrt{q+H}}}{q-H_{1}}-a_{5} \frac{\sqrt{q} e^{-\xi \sqrt{q+H}}}{q-H_{1}} \\
& -a_{1} \frac{e^{-\xi \sqrt{q a}}}{\sqrt{q}-d}-a_{2} \frac{e^{-\xi \sqrt{q a}}}{q}-a_{3} \frac{e^{-\xi \sqrt{q a}}}{\sqrt{q}} \\
& +a_{4} \frac{e^{-\xi \sqrt{q a}}}{q-H_{1}}+a_{5} \frac{\sqrt{q} e^{-\xi \sqrt{q a}}}{q-H_{1}} .
\end{aligned}
$$


The solution of (16), after taking the Laplace inverse, is given by

$$
\begin{aligned}
v_{0}(\xi, \tau)= & f_{1}(\xi, \tau, H)+a_{2} f_{1}(\xi, \tau, H)-a_{2} f_{1}(\xi, \tau, 0) \\
& -a_{4} e^{H_{1} t} f_{1}\left(\xi, \tau, H+H_{1}\right)+a_{4} e^{H_{1} t} f_{1}\left(\xi \sqrt{a}, \tau, H_{1}\right) \\
& -a_{1} f_{2}(\xi \sqrt{a}, \tau, d)+2 f_{6}(\xi, z, \tau, H) \\
& +2 d f_{7}(\xi, z, \tau, H)+2 f_{8}(\xi, z, \tau, H) \\
& -2 f_{9}(\xi, z, \tau, H)-2 H_{1} f_{10}(\xi, z, \tau, H) \\
& -f_{11}(\xi, z, \tau, H)+2 f_{12}(\xi, z, \tau, H) \\
& +2 H_{1} f_{13}(\xi, z, \tau, H) .
\end{aligned}
$$

\section{Flow due to Uniform Acceleration of the Plate}

For the uniform acceleration of the plate $(n=1),(12)$ yields

$$
\begin{aligned}
\bar{v}_{1}(\xi, q)= & \frac{1}{q^{2}} e^{-\xi \sqrt{q+H}}+a_{1} \frac{e^{-\xi \sqrt{q+H}}}{\sqrt{q}-d}+a_{2} \frac{e^{-\xi \sqrt{q+H}}}{q} \\
& +a_{3} \frac{e^{-\xi \sqrt{q+H}}}{\sqrt{q}}-a_{4} \frac{e^{-\xi \sqrt{q+H}}}{q-H_{1}} \\
& -a_{5} \frac{\sqrt{q} e^{-\xi \sqrt{q+H}}}{q-H_{1}}-a_{1} \frac{e^{-\xi \sqrt{q a}}}{\sqrt{q}-d} \\
& -a_{2} \frac{e^{-\xi \sqrt{q a}}}{q}-a_{3} \frac{e^{-\xi \sqrt{q a}}}{\sqrt{q}}+a_{4} \frac{e^{-\xi \sqrt{q a}}}{q-H_{1}} \\
& +a_{5} \frac{\sqrt{q} e^{-\xi \sqrt{q a}}}{q-H_{1}} .
\end{aligned}
$$

The solution of (18), after taking the Laplace inverse, is given by

$$
\begin{aligned}
v_{1}(\xi, \tau)= & a_{2} f_{1}(\xi, \tau, H)-a_{2} f_{1}(\xi \sqrt{a}, \tau, 0) \\
& -a_{4} e^{H_{1} t} f_{1}\left(\xi, \tau, H+H_{1}\right)+a_{4} e^{H_{1} t} f_{1}\left(\xi \sqrt{a}, \tau, H_{1}\right) \\
& -a_{1} f_{2}(\xi \sqrt{a}, \tau, d)+f_{3}(\xi, \tau, H)+2 f_{6}(\xi, z, \tau, H) \\
& +2 d f_{7}(\xi, z, \tau, H)+2 f_{8}(\xi, z, \tau, H) \\
& -2 f_{9}(\xi, z, \tau, H)-2 H_{1} f_{10}(\xi, z, \tau, H) \\
& -f_{11}(\xi, z, \tau, H)+2 f_{12}(\xi, z, \tau, H) \\
& +2 H_{1} f_{13}(\xi, z, \tau, H) .
\end{aligned}
$$

\section{Flow due to Nonuniform Acceleration of the Plate}

For the nonuniform acceleration of the plate $(n=2),(12)$ yields

$$
\begin{aligned}
\bar{v}_{2}(\xi, q)= & \frac{1}{q^{3}} e^{-\xi \sqrt{q+H}}+a_{1} \frac{e^{-\xi \sqrt{q+H}}}{\sqrt{q}-d}+a_{2} \frac{e^{-\xi \sqrt{q+H}}}{q} \\
& +a_{3} \frac{e^{-\xi \sqrt{q+H}}}{\sqrt{q}}-a_{4} \frac{e^{-\xi \sqrt{q+H}}}{q-H_{1}}-a_{5} \frac{\sqrt{q} e^{-\xi \sqrt{q+H}}}{q-H_{1}} \\
& -a_{1} \frac{e^{-\xi \sqrt{q a}}}{\sqrt{q}-d}-a_{2} \frac{e^{-\xi \sqrt{q a}}}{q}-a_{3} \frac{e^{-\xi \sqrt{q a}}}{\sqrt{q}} \\
& +a_{4} \frac{e^{-\xi \sqrt{q a}}}{q-H_{1}}+a_{5} \frac{\sqrt{q} e^{-\xi \sqrt{q a}}}{q-H_{1}} .
\end{aligned}
$$

The solution of (20), after taking the Laplace inverse, is given by

$$
\begin{aligned}
v_{2}(\xi, \tau)= & a_{2} f_{1}(\xi, \tau, H)-a_{2} f_{1}(\xi \sqrt{a}, \tau, 0) \\
& -a_{4} e^{H_{1} t} f_{1}\left(\xi, \tau, H+H_{1}\right) \\
& +a_{4} e^{H_{1} t} f_{1}\left(\xi \sqrt{a}, \tau, H_{1}\right) \\
& -a_{1} f_{2}(\xi \sqrt{a}, \tau, d)+f_{4}(\xi, \tau, H) \\
& +2 f_{6}(\xi, z, \tau, H)+2 d f_{7}(\xi, z, \tau, H) \\
& +2 f_{8}(\xi, z, \tau, H)-2 f_{9}(\xi, z, \tau, H) \\
& -2 H_{1} f_{10}(\xi, z, \tau, H)-f_{11}(\xi, z, \tau, H) \\
& +2 f_{12}(\xi, z, \tau, H)+2 H_{1} f_{13}(\xi, z, \tau, H)
\end{aligned}
$$

\section{Flow due to Highly Nonuniform Acceleration of the Plate}

In this case, $n=3$ and (12) yields

$$
\begin{aligned}
\bar{v}_{3}(\xi, q)= & \frac{1}{q^{4}} e^{-\xi \sqrt{q+H}}+a_{1} \frac{e^{-\xi \sqrt{q+H}}}{\sqrt{q}-d}+a_{2} \frac{e^{-\xi \sqrt{q+H}}}{q} \\
& +a_{3} \frac{e^{-\xi \sqrt{q+H}}}{\sqrt{q}}-a_{4} \frac{e^{-\xi \sqrt{q+H}}}{q-H_{1}}-a_{5} \frac{\sqrt{q} e^{-\xi \sqrt{q+H}}}{q-H_{1}} \\
& -a_{1} \frac{e^{-\xi \sqrt{q a}}}{\sqrt{q}-d}-a_{2} \frac{e^{-\xi \sqrt{q a}}}{q}-a_{3} \frac{e^{-\xi \sqrt{q a}}}{\sqrt{q}} \\
& +a_{4} \frac{e^{-\xi \sqrt{q a}}}{q-H_{1}}+a_{5} \frac{\sqrt{q} e^{-\xi \sqrt{q a}}}{q-H_{1}} .
\end{aligned}
$$


The solution of (22), after taking the Laplace inverse, is given by

$$
\begin{aligned}
v_{3}(\xi, \tau)= & a_{2} f_{1}(\xi, \tau, H)-a_{2} f_{1}(\xi \sqrt{a}, \tau, 0) \\
& -a_{4} e^{H_{1} t} f_{1}\left(\xi, \tau, H+H_{1}\right)+a_{4} e^{H_{1} t} f_{1}\left(\xi \sqrt{a}, \tau, H_{1}\right) \\
& -a_{1} f_{2}(\xi \sqrt{a}, \tau, d)+f_{5}(\xi, \tau, H)+2 f_{6}(\xi, z, \tau, H) \\
& +2 d f_{7}(\xi, z, \tau, H)+2 f_{8}(\xi, z, \tau, H) \\
& -2 f_{9}(\xi, z, \tau, H)-2 H_{1} f_{10}(\xi, z, \tau, H) \\
& -f_{11}(\xi, z, \tau, H)+2 f_{12}+2 H_{1} f_{13},
\end{aligned}
$$

where

$$
\begin{aligned}
& f_{1}(y, t, w)=\frac{1}{2}\left(e^{-y \sqrt{w}} \operatorname{erf} c\left[\frac{y}{2 \sqrt{t}}-\sqrt{w t}\right]\right. \\
& \left.+e^{y \sqrt{w}} \operatorname{erf} c\left[\frac{y}{2 \sqrt{t}}+\sqrt{w t}\right]\right) \\
& f_{2}(y, t, w)=\frac{1}{\sqrt{\pi t}} \exp \left(-\frac{y^{2}}{4 t}\right) \\
& +w \exp \left(-y w+w^{2} t\right) \operatorname{erf} c\left[\frac{y}{2 \sqrt{t}}-w \sqrt{t}\right], \\
& f_{3}(y, t, w)=\frac{1}{2}\left(\left(t-\frac{y}{2 \sqrt{w}}\right) e^{-\xi \sqrt{w}} \operatorname{erf} c\left[\frac{y}{2 \sqrt{t}}-\sqrt{w t}\right]\right. \\
& +\left(t+\frac{y}{2 \sqrt{w}}\right) e^{y \sqrt{w}} \\
& \left.\times \operatorname{erf} c\left[\frac{y}{2 \sqrt{t}}+\sqrt{w t}\right]\right), \\
& f_{4}(y, t, w)=\frac{1}{4}\left(\left(t^{2}-\frac{y t}{\sqrt{w}}+\frac{y^{2}}{4 w}+\frac{y}{4 w \sqrt{w}}\right)\right. \\
& \left.\times e^{-y \sqrt{w}} \operatorname{erf} c\left[\frac{y}{2 \sqrt{t}}-\sqrt{w t}\right]\right) \\
& +\left(t^{2}+\frac{y t}{\sqrt{w}}+\frac{y^{2}}{4 w}-\frac{y}{4 w \sqrt{w}}\right) \\
& \left.\times e^{y \sqrt{w}} \operatorname{erf} c\left[\frac{y}{2 \sqrt{t}}+\sqrt{w t}\right]\right), \\
& f_{5}(y, t, w)=\frac{1}{6}\left(\frac{3 y}{4 w^{2}}-\frac{y t}{w}\right) \sqrt{\frac{t}{w}} \exp \left(\frac{y^{2}}{4 t}-w t\right) \\
& +\left(-\frac{y^{2}}{32 w^{2}}-\frac{y^{2} t}{16 w}+\frac{t^{3}}{12}\right) \\
& \times e^{-y \sqrt{w}} \operatorname{erf} c\left[\frac{y}{2 \sqrt{t}}-\sqrt{w t}\right]
\end{aligned}
$$

$$
\begin{gathered}
+e^{y \sqrt{w}} \operatorname{erf} c\left[\frac{y}{2 \sqrt{t}}+\sqrt{w t}\right] \\
\times\left(-\frac{y}{32 w^{2} \sqrt{w}}-\frac{y^{2}}{96 w \sqrt{w}}\right. \\
\left.+\frac{y t}{16 w \sqrt{w}}-\frac{y t^{2}}{8 \sqrt{w}}\right) \\
\times\left(e^{-y \sqrt{w}} \operatorname{erf} c\left[\frac{y}{2 \sqrt{t}}-\sqrt{w t}\right]\right. \\
\left.+e^{y \sqrt{w}} \operatorname{erf} c\left[\frac{y}{2 \sqrt{t}}+\sqrt{w t}\right]\right),
\end{gathered}
$$

$f_{6}(\xi, z, \tau, H)$

$$
\begin{aligned}
& =\frac{a_{1}}{\pi} \int_{\xi / 2 \sqrt{\tau}}^{\infty} \frac{\exp \left(-z^{2}-H\left(\xi^{2} / 4 z^{2}\right)\right)}{\sqrt{\tau-\left(\xi^{2} / 4 z^{2}\right)}} d z, \\
& f_{7}(\xi, z, \tau, H)=\frac{a_{1}}{\sqrt{\pi}} \int_{(\xi / 2 \sqrt{\tau})}^{\infty} \exp \left(-z^{2}-H \frac{\xi^{2}}{4 z^{2}}\right. \\
& \left.+d^{2} \tau-d^{2} \frac{\xi^{2}}{4 z^{2}}\right) \\
& \times \operatorname{erf}\left[-d \sqrt{\tau-\frac{\xi^{2}}{4 z^{2}}}\right] d z \\
& f_{8}(\xi, z, \tau, H)=\frac{a_{3}}{\pi} \int_{\xi / 2 \sqrt{\tau}}^{\infty} \frac{\exp \left(-z^{2}-H\left(\xi^{2} / 4 z^{2}\right)\right)}{\sqrt{\tau-\left(\xi^{2} / 4 z^{2}\right)}} d z \\
& f_{9}(\xi, z, \tau, H)=\frac{a_{5}}{\pi} \int_{\xi / 2 \sqrt{\tau}}^{\infty} \frac{\exp \left(-z^{2}-H\left(\xi^{2} / 4 z^{2}\right)\right)}{\sqrt{\tau-\left(\xi^{2} / 4 z^{2}\right)}} d z \\
& f_{10}(\xi, z, \tau, H)=\frac{a_{5}}{\sqrt{\pi}} \int_{\xi / 2 \sqrt{\tau}}^{\infty} \exp \left(-z^{2}-H \frac{\xi^{2}}{4 z^{2}}\right. \\
& \left.+H_{1} \tau-H_{1} \frac{\xi^{2}}{4 z^{2}}\right) \\
& \times \operatorname{erf}\left[\sqrt{H_{1}\left(\tau-\frac{\xi^{2}}{4 z^{2}}\right)}\right] d z \\
& f_{11}(\xi, z, \tau, H)=\exp \left(\frac{\xi^{2} a}{4 \tau}\right) \text {, } \\
& f_{12}(\xi, z, \tau, H)=\frac{a_{5} \sqrt{a}}{\pi} \int_{\xi / 2 \sqrt{\tau}}^{\infty} \frac{\exp \left(-z^{2} a\right)}{\sqrt{\tau-\left(\xi^{2} / 4 z^{2}\right)}} d z, \\
& f_{13}(\xi, z, \tau, H)=\frac{a_{5} \sqrt{a}}{\sqrt{\pi}} \int_{\xi / 2 \sqrt{\tau}}^{\infty} \exp \left(-z^{2} a+H_{1} \tau\right.
\end{aligned}
$$




$$
\begin{aligned}
& \left.-H_{1} \frac{\xi^{2}}{4 z^{2}}\right) \\
& \times \operatorname{erf}\left[\sqrt{H_{1}\left(\tau-\frac{\xi^{2}}{4 z^{2}}\right)}\right] d z
\end{aligned}
$$

\section{Special Cases}

The following solutions from the literature appear as the limiting cases of our general solutions.

\subsection{Absence of Thermal Effects}

7.1.1. Case I: Impulsive Motion of the Plate. By eliminating the convectional term $\mathrm{Gr}=0$ together with $\mathrm{MHD}$ and porosity effects $\left(M=0\right.$ and $K \rightarrow \infty$; that is, $\left.H=\beta_{0}\right)$ in (17), we get

$$
v_{0}=f_{1}\left(\xi, \tau, \beta_{0}\right) \text {, }
$$

which is in quite agreement with [40, Equation (2.7)] when $v=U=1$. Furthermore by putting $\beta_{0}=0$, (25) reduces to

$$
v_{0 N}=f_{1}(\xi, \tau, 0),
$$

which is the well-known solution of Newtonian fluid [40, Equation (2.8)] for the impulsive motion of the plate obtained by a different technique.

7.1.2. Case II: Accelerated Motion of the Plate. In this case when convectional term $\mathrm{Gr}=0$ with $M=0$ and $K \rightarrow \infty$, (19) reduces to

$$
v_{1}=f_{3}\left(\xi, \tau, \beta_{1}\right),
$$

which is in complete agreement with [41, Equation (15)] for accelerated motion of the plate.

Skin Friction. The expressions for skin frictions $\tau_{0}$ and $\tau_{1}$ evaluated from (17) and (19), respectively, are given by

$$
\begin{aligned}
\tau_{0}=-\left.\frac{\partial v_{0}(\xi, \tau)}{\partial \xi}\right|_{\xi=0}= & -g_{1}(\tau, H)-a_{2} g_{1}(\tau, H) \\
& +a_{2} g_{1}(\tau, 0)+a_{4} e^{H_{1} \tau} g_{1}\left(\tau, H+H_{1}\right) \\
& -a_{4} \sqrt{a} e^{H_{1} \tau} g_{1}\left(\tau, H_{1}\right) \\
& +a_{1} \sqrt{a} g_{2}(\tau, d), \\
\tau_{1}=-\left.\frac{\partial v_{1}(\xi, \tau)}{\partial \xi}\right|_{\xi=0}= & -a_{2} g_{1}(\tau, H)+a_{2} g_{1}(\tau, 0) \\
& +a_{4} e^{H_{1} \tau} g_{1}\left(\tau, H+H_{1}\right) \\
& -a_{4} \sqrt{a} e^{H_{1} \tau} g_{1}\left(\tau, H_{1}\right) \\
& +a_{1} \sqrt{a} g_{2}(\tau, d)-g_{3}(\tau, H),
\end{aligned}
$$

where

$$
\begin{gathered}
g_{1}(t, w)=\left.\frac{\partial f_{1}}{\partial y}(y, t, w)\right|_{y=0} \\
=-\frac{e^{-w t}\left(1+e^{w t} \sqrt{w} \sqrt{\pi t} \operatorname{erf}[\sqrt{w t}]\right)}{\sqrt{\pi t}}, \\
g_{2}(t, w) \\
=\left.\frac{\partial f_{2}}{\partial y}(y, t, w)\right|_{y=0} \\
=\frac{a_{1} \sqrt{a} d\left(-1-2 d e^{d^{2} t} \sqrt{\pi t}+d e^{d^{2} t} \sqrt{\pi t} \operatorname{erf} c[d \sqrt{t}]\right)}{\sqrt{\pi t}}, \\
g_{3}(t, w)=\left.\frac{\partial f_{3}}{\partial y}(y, t, w)\right|_{y=0} \\
=-\frac{e^{-w t} \sqrt{t}}{\sqrt{\pi}}-\frac{(1+2 t w) \operatorname{erf}[\sqrt{w t}]}{2 \sqrt{w}} .
\end{gathered}
$$

Nusselt Number. The rate of heat transfer evaluated from (15) is given by

$$
\begin{aligned}
\mathrm{Nu} & =-\left.\frac{\partial \theta(\xi, \tau)}{\partial \xi}\right|_{\xi=0}=\gamma\left[\frac{1}{\theta(0, \tau)}+1\right] \\
& =\gamma\left[\frac{1}{e^{d^{2} \tau}(1+\operatorname{erf}[d \sqrt{\tau}])-1}+1\right] .
\end{aligned}
$$

\section{Graphical Results and Discussion}

In this paper, the influence of thermal radiation on unsteady free convection flow of MHD Brinkman type fluid past a vertical plate in a porous medium is studied with Newtonian heating condition. Four different types of motions in terms of different boundary conditions on velocity are discussed. Exact solutions are obtained by means of Laplace transforms. Straightforward computations show that the velocities $v_{0}(y, t), v_{1}(y, t), v_{2}(y, t)$, and $v_{3}(y, t)$ given by (17), (19), (21), and (23) and temperature (15), respectively, satisfy both the governing equations and all imposed initial and boundary conditions. As a special case when $\beta_{n}(n=$ $0-3) \rightarrow 0$, the solutions that have been established reduce to the well-known solutions for Newtonian fluids. It is worthy pointing out that solutions corresponding to hydrodynamic motion of Brinkman fluid in a nonporous medium can also be obtained as a limiting case. In order to study the physical aspects of the problem, the analytical results for velocities $v_{0}(\xi, \tau)$ and $v_{1}(\xi, \tau)$ due to impulsive motion and uniform acceleration as well as for temperature distribution are plotted graphically in Figures 2-12, whereas the numerical results of skin friction and Nusselt number for different flow parameters are shown in Tables 1 and 2. The parameters entering into the problem are Prandtl number Pr, magnetic 


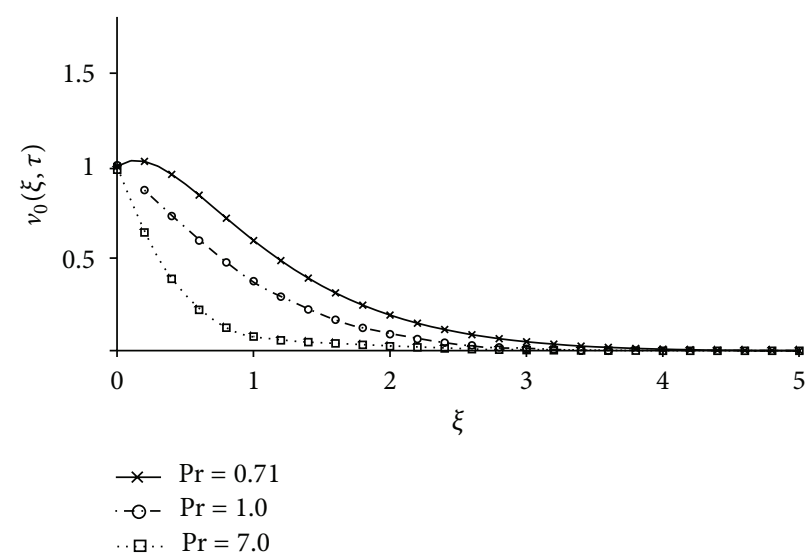

(a)

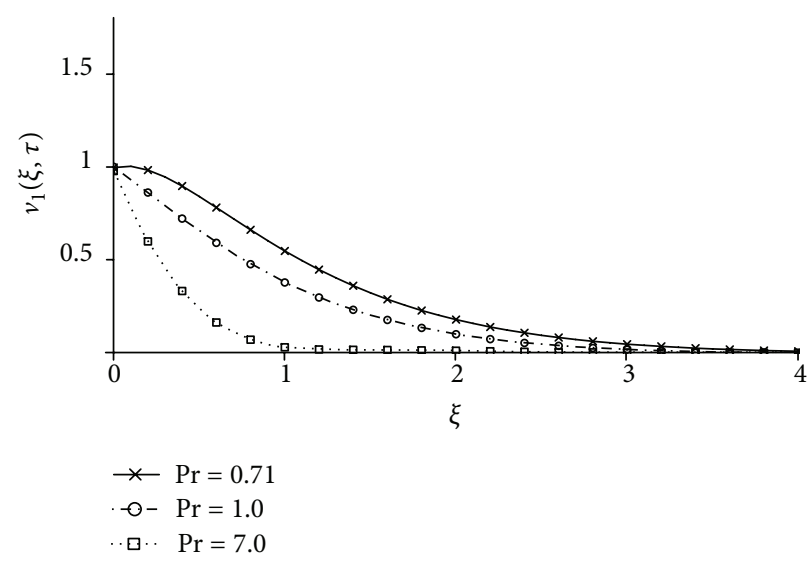

(b)

FIgURE 2: Velocity profiles for different values of $\operatorname{Pr}$ where $M=2, K=1, \mathrm{Gr}=0.5, R=1, \gamma=1, \beta_{0}=\beta_{1}=1$, and $\tau=1$.

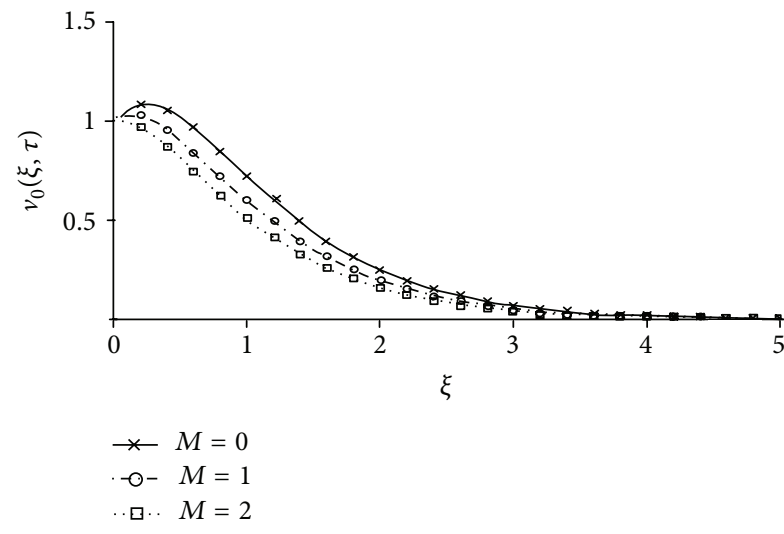

(a)

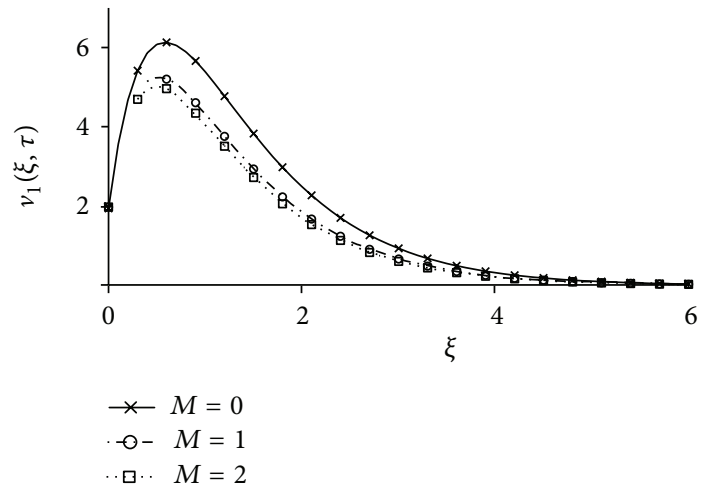

(b)

FIgURE 3: Velocity profiles for different values of $M$ where $\operatorname{Pr}=0.71, K=1, \mathrm{Gr}=0.5, R=1, \gamma=1, \beta_{0}=\beta_{1}=1$, and $\tau=1$.

TABLE 1: Variation in skin friction $\tau_{0}$ and $\tau_{1}$.

\begin{tabular}{lcccccccccc}
\hline $\operatorname{Pr}$ & $M$ & $K$ & $\mathrm{Gr}$ & $R$ & $\gamma$ & $\beta_{0}$ & $\beta_{1}$ & $\tau$ & $\tau_{0}$ & $\tau_{1}$ \\
\hline 0.71 & 1 & 1 & 0.5 & 1 & 0.2 & 1 & 1 & 1 & 2.029 & 2.314 \\
$\mathbf{1}$ & 1 & 1 & 0.5 & 1 & 0.2 & 1 & 1 & 1 & 2.035 & 2.320 \\
0.71 & $\mathbf{2}$ & 1 & 0.5 & 1 & 0.2 & 1 & 1 & 1 & 2.656 & 2.860 \\
0.71 & 1 & $\mathbf{2}$ & 0.5 & 1 & 0.2 & 1 & 1 & 1 & 1.910 & 2.218 \\
0.71 & 1 & 1 & $\mathbf{1}$ & 1 & 0.2 & 1 & 1 & 1 & 2.324 & 2.608 \\
0.71 & 1 & 1 & 0.5 & $\mathbf{2}$ & 0.2 & 1 & 1 & 1 & 2.025 & 2.310 \\
0.71 & 1 & 1 & 0.5 & 1 & $\mathbf{0 . 4}$ & 1 & 1 & 1 & 2.089 & 2.373 \\
0.71 & 1 & 1 & 0.5 & 1 & 0.2 & $\mathbf{2}$ & $\mathbf{2}$ & 1 & 2.255 & 2.504 \\
0.71 & 1 & 1 & 0.5 & 1 & 0.2 & 1 & 1 & $\mathbf{2}$ & 2.038 & 4.059 \\
\hline
\end{tabular}

The bold values show the comparison (increase or decrease) with the fixed values (not bold) of the same column for the corresponding parameters given at the top of the column on the skin frictions $\left(\tau_{0}\right.$ and $\left.\tau_{1}\right)$.

parameter $M$, porosity parameter $K$, Grashof number $\mathrm{Gr}$, radiation parameter $R$, Newtonian heating parameter $\gamma$, dimensionless time $\tau$, and Brinkman parameter $\beta_{n}(n=$ $0,1)$.
TABLE 2: Variation in Nusselt number Nu.

\begin{tabular}{llllc}
\hline $\operatorname{Pr}$ & $R$ & $\gamma$ & $\tau$ & $\mathrm{Nu}$ \\
\hline 0.71 & 1 & 0.2 & 1 & 0.305 \\
7 & 1 & 0.2 & 1 & 0.284 \\
0.71 & 2 & 0.2 & 1 & 0.340 \\
0.71 & 1 & $\mathbf{0 . 4}$ & 1 & 1.040 \\
0.71 & 1 & 0.2 & 2 & 0.375 \\
\hline
\end{tabular}

The bold values show the comparison (increase or decrease) with the fixed values (not bold) of the same column for the corresponding parameters given at the top of the column on Nusselt number $(\mathrm{Nu})$.

Figure 2 is plotted to see the effect of Pr on velocity in cases of impulsive motion $v_{0}(\xi, \tau)$ and uniform acceleration $v_{1}(\xi, \tau)$ of the plate. Three different values of $\operatorname{Pr}=0.71,1$, and 7 are chosen such that physically they correspond to air, electrolytic solution, and water, respectively. It is clear from this figure that velocity decreases for both impulsive motion and accelerated motion of the plate as Pr increases. Physically, it is true due to the fact that an increase in Prandtl number Pr increases viscosity of 


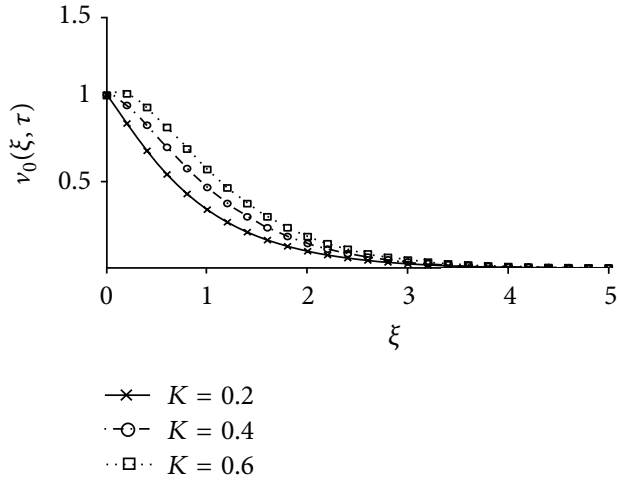

(a)

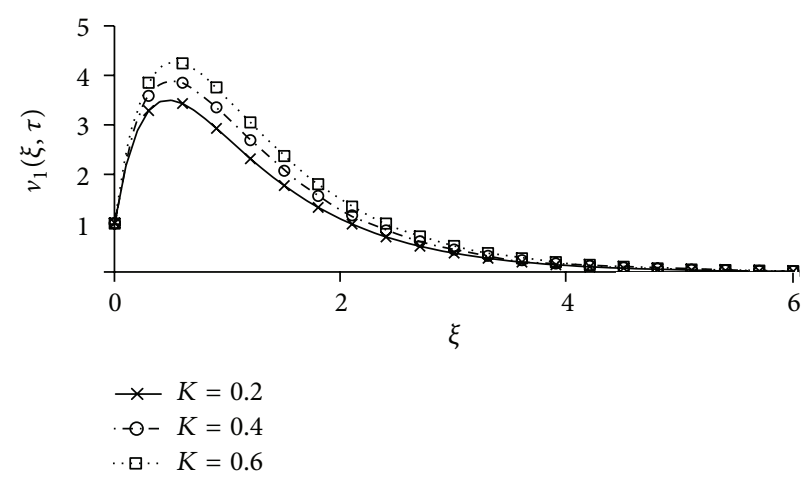

(b)

FIgURE 4: Velocity profiles for different values of $K$ where $M=2, \operatorname{Pr}=0.71, \mathrm{Gr}=0.5, R=1, \gamma=1, \beta_{0}=\beta_{1}=1$, and $\tau=1$.

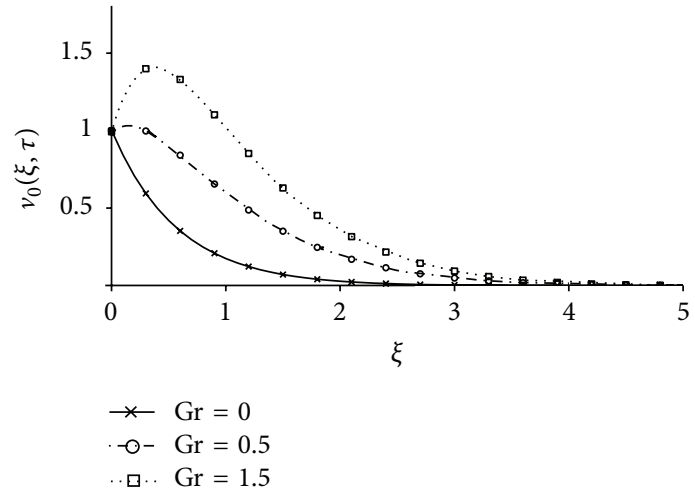

(a)

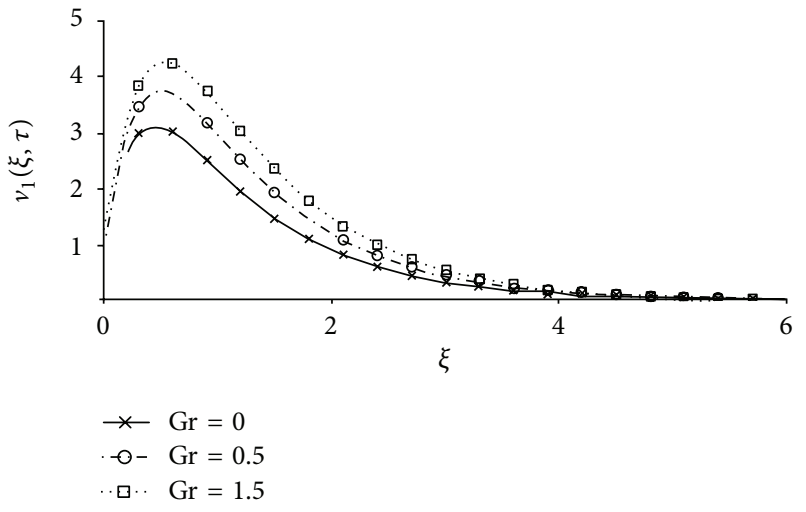

(b)

FIGURE 5: Velocity profiles for different values of Gr where $M=2, K=1, \operatorname{Pr}=0.71, R=1, \gamma=1, \beta_{0}=\beta_{1}=1$, and $\tau=1$.

the fluid. Hence, the fluid becomes thick and consequently velocity decreases. Figure 3 is prepared to see the effects of the magnetic parameter $M$ on velocity profiles for impulsive and accelerated motions of the plate. It is observed that velocity in both cases decreases when $M$ is increased. This physical interpretation of the velocity is an indication that the increasing values of magnetic parameter $M$ make the resistive forces strong enough so that they can oppose the fluid motion and as a result velocity decreases. This figure also shows the comparison of hydrodynamic velocity to hydromagnetic velocity of Brinkman fluid. In the absence of magnetic effects the velocity is maximum. Furthermore, it is observed that the momentum boundary layer thickness for the impulsive motion is greater compared to uniformly accelerated motion. However, the magnitude of velocity in case of uniformly accelerated motion is greater than that of impulsive motion.

Figure 4 is plotted to show the effects of porosity parameter $K$ on the velocities $v_{0}(\xi, \tau)$ and $v_{1}(\xi, \tau)$. It appears from this figure that with increasing values of $K$, velocity increases for both impulsive and uniformly accelerated motions. Physically, it is true because increasing $K$ reduces the drag force and causes the velocity profiles to increase. Thus increasingvalue of the porosity parameter $K$ yields an effect opposite to that of the magnetic parameter $M$. Figure 5 shows the effect of Grashof number Gr on the velocities for the cases of impulsive and uniformly accelerated motions. It is observed that an increase in Grashof number Gr leads to increasing both types of velocities $v_{0}(\xi, \tau)$ and $v_{1}(\xi, \tau)$ due to enhancement in buoyancy force. It is true because thermal Grashof number signifies the relative effect of thermal buoyancy force to viscous hydrodynamic force. Increase of Grashof number Gr means increase of temperature gradient $\left(T_{w}-T_{\infty}\right)$ due to which the contribution from the buoyancy near the plate becomes significant and hence a short rise in the velocity near the plate is observed. Physically, Gr = 0 corresponds to the absence of free convection current and $\mathrm{Gr}>0$ means heating of the fluid or cooling of the plate by natural convection. For the positive values of $\mathrm{Gr}$, heat is conducted away from the vertical plate into the fluid which increases the temperature and thereby enhances the buoyancy force.

The graphs showing the variations of radiation parameter $R$ on the velocities $v_{0}(\xi, \tau)$ and $v_{1}(\xi, \tau)$ are displayed in Figure 6 . We found that in both cases the velocity increases as $R$ increases. Physically, it is true, as higher radiation occurs when temperature is higher and ultimately velocity 


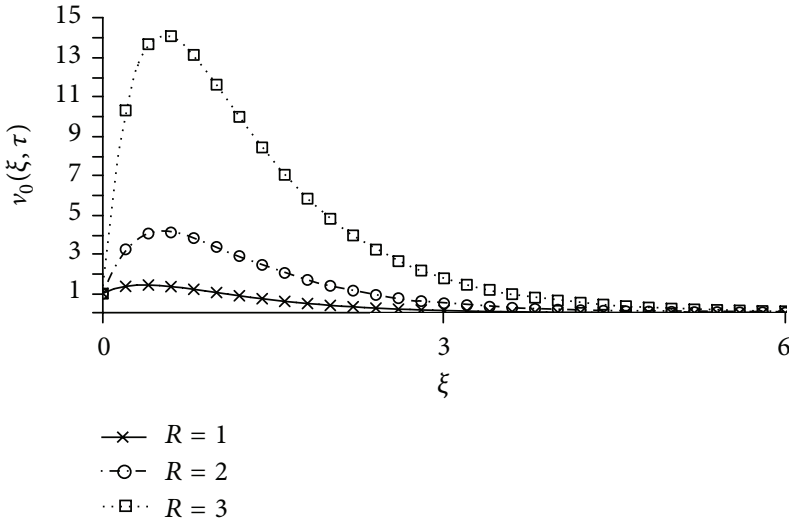

(a)

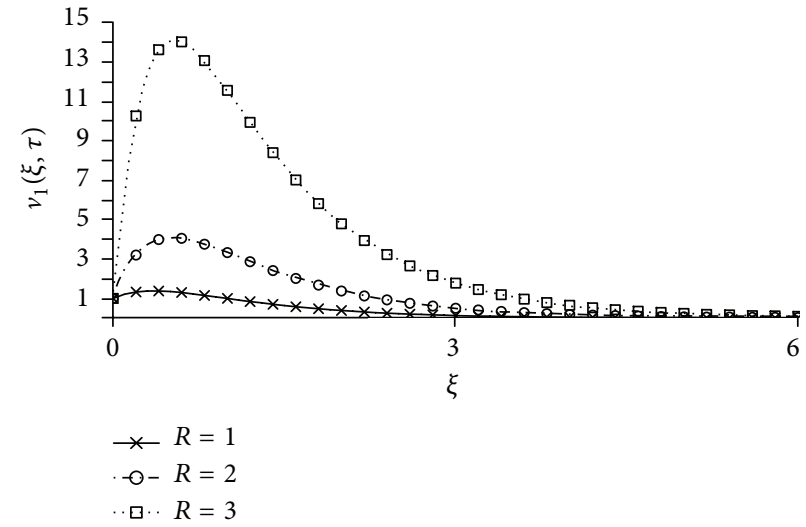

(b)

Figure 6: Velocity profiles for different values of $R$ where $M=2, K=1, \mathrm{Gr}=0.5, \operatorname{Pr}=0.71, \gamma=1, \beta_{0}=\beta_{1}=1$, and $\tau=1$.

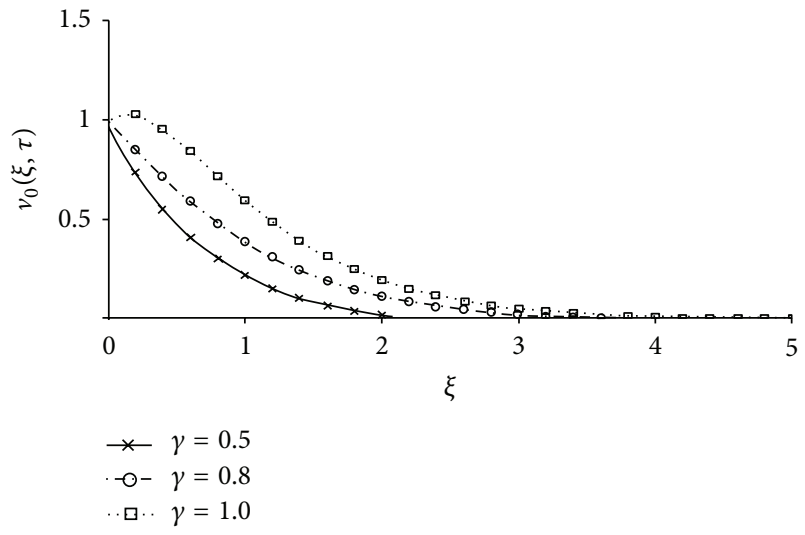

(a)

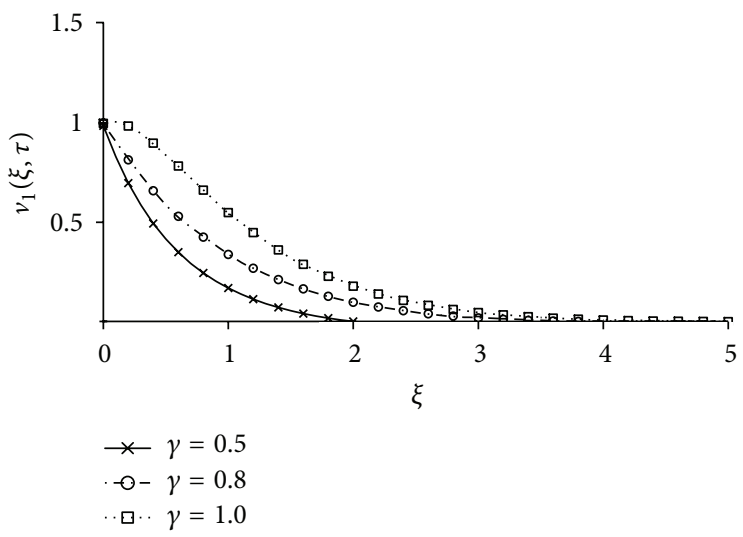

(b)

FIGURE 7: Velocity profiles for different values of $\gamma$ where $M=2, K=1, \mathrm{Gr}=0.5, R=1, \operatorname{Pr}=0.71, \beta_{0}=\beta_{1}=1$, and $\tau=1$.

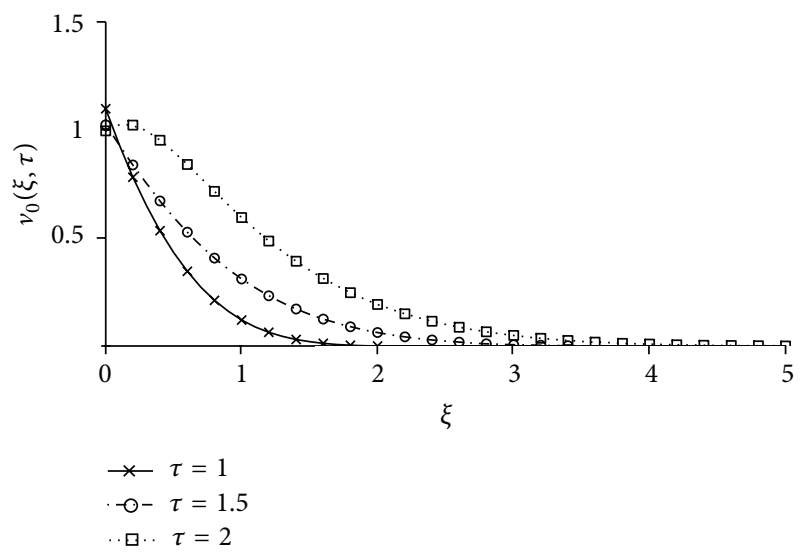

(a)

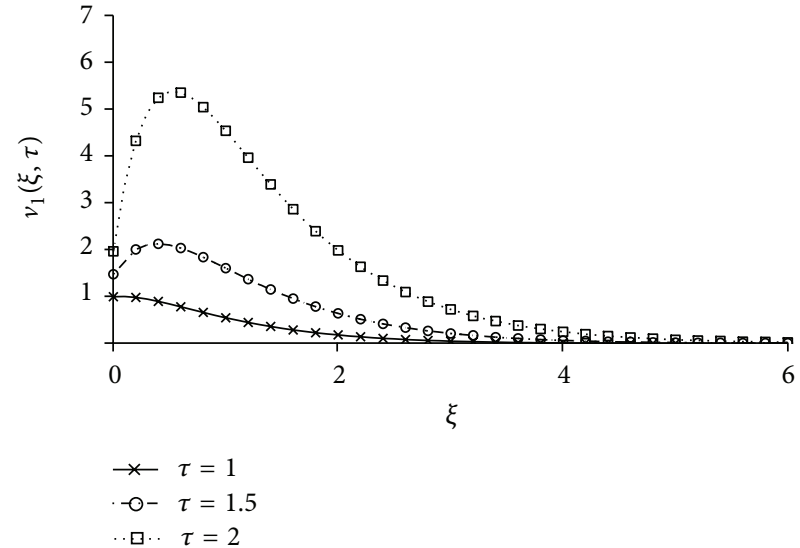

(b)

FIGURE 8: Velocity profiles for different values of $\tau$ where $M=2, K=1, \mathrm{Gr}=0.5, R=1, \gamma=1, \beta_{0}=\beta_{1}=1$, and $\operatorname{Pr}=0.71$. 


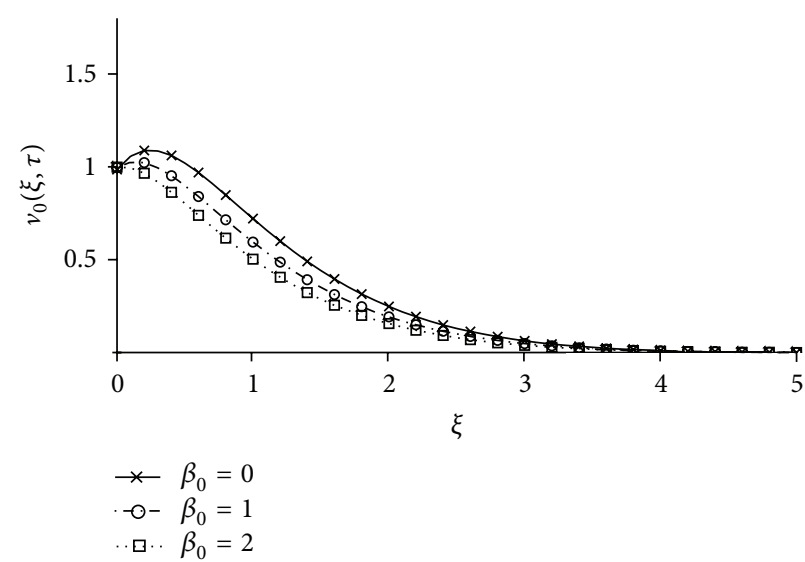

(a)

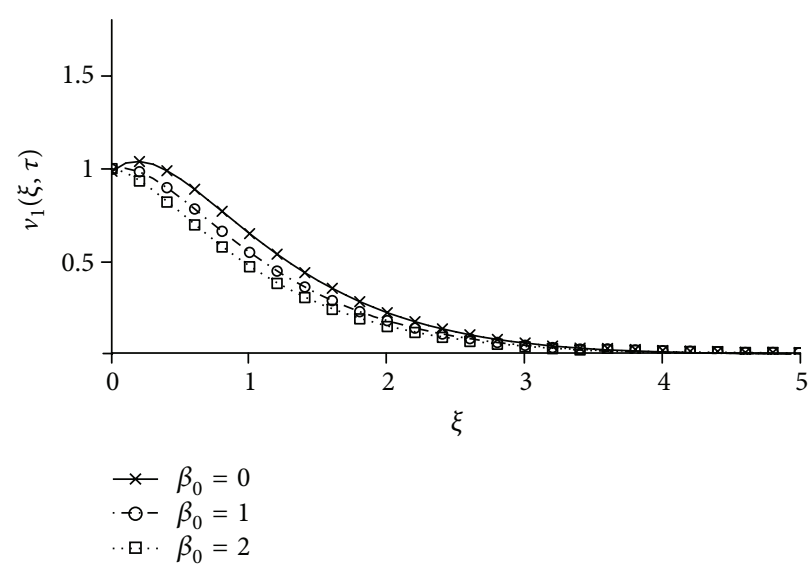

(b)

FIGURE 9: Velocity profiles for different values of $\beta_{0}$ and $\beta_{1}$ where $M=2, K=1, \mathrm{Gr}=0.5, R=1, \gamma=1, \operatorname{Pr}=0.71$, and $\tau=1$.

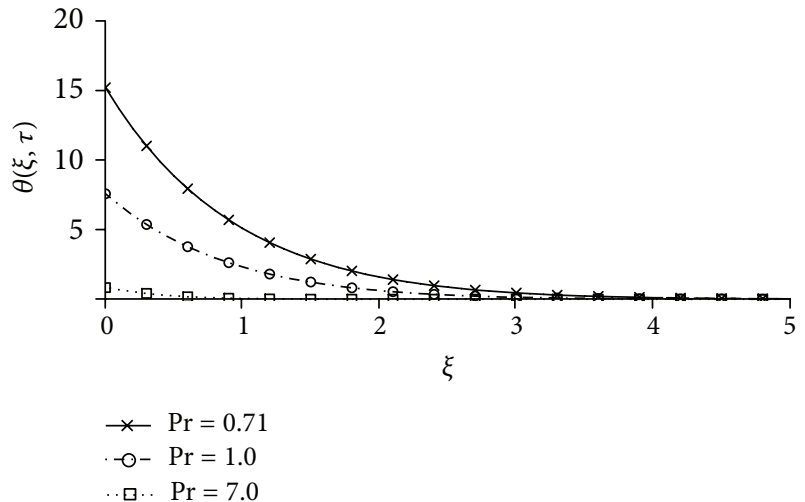

FIGURE 10: Temperature profiles for different values of Pr where $R=$ $1, \gamma=1$, and $\tau=1$

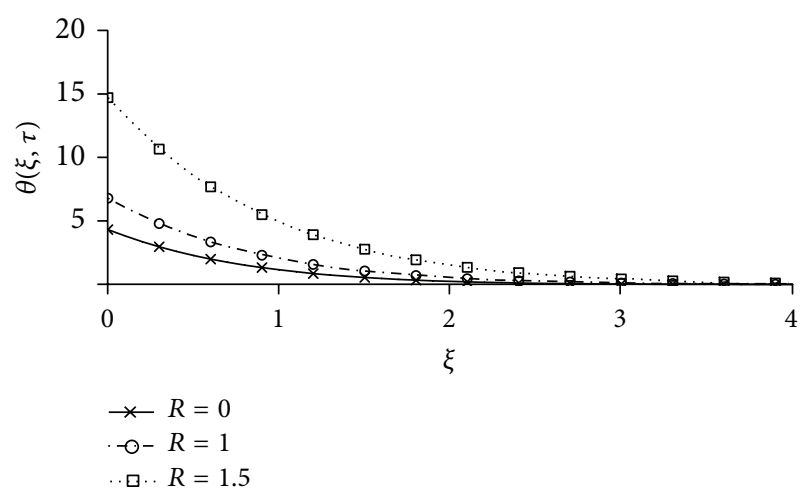

FIGURE 11: Temperature profiles for different values of $R$ where $\operatorname{Pr}=$ $0.71, \gamma=1$, and $\tau=1$.

rises. Figure 7 is plotted to show the influence of conjugate parameter $\gamma$ on velocities $v_{0}(\xi, \tau)$ and $v_{1}(\xi, \tau)$. We observe that velocity increases in both figures as $\gamma$ increases. The influence of dimensionless time $\tau$ on velocity is shown in Figure 8. It is seen from this figure that velocity

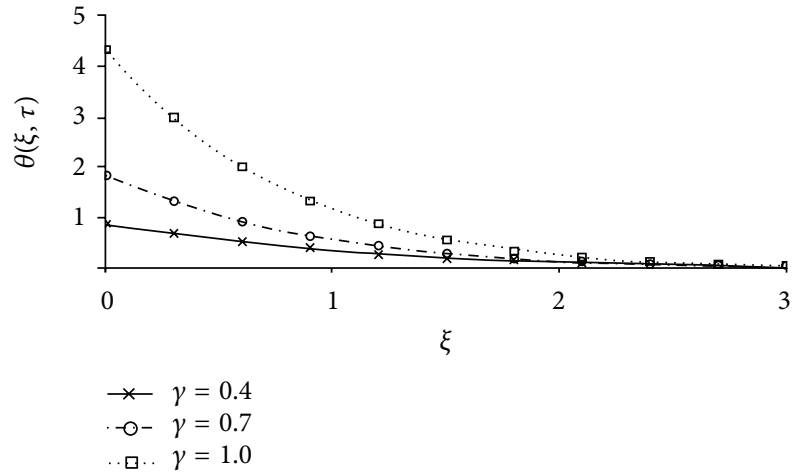

FIgURE 12: Temperature profiles for different values of $\gamma$ where $R=$ $1, \operatorname{Pr}=0.71$, and $\tau=1$.

increases when $\tau$ increases. Furthermore, both of these velocities $v_{0}(\xi, \tau)$ and $v_{1}(\xi, \tau)$ satisfy the given boundary conditions (see (11)). This proves the accuracy of our obtained results. The effects of Brinkman parameter $\beta_{n}(n=0,1)$ on the flow due to impulsive and uniformly accelerated motions of the plate are studied in Figure 9. We observe that velocity decreases as $\beta$ increases. Physically, it is true in the sense that the Brinkman type fluid can move slowly due to the drag properties of the fluid.

Figure 10 is sketched to show the effects of Prandtl number $\operatorname{Pr}$ on temperature profiles. Three different values of $\operatorname{Pr}=0.71,1$, and 7 that physically correspond to air, electrolytic solution, and water are chosen. It is observed that increasing values of Pr lead to a fall in the temperature. A similar behavior was also expected due to the fact that at smaller values of Pr fluids possess high thermal conductivity and heat diffuses away from the surface faster than at higher values of Pr. Thus the boundary layer becomes thicker and consequently temperature decreases when $\mathrm{Pr}$ is increased.

Figure 11 is plotted to show the influence of radiation parameter $R$ on the temperature profiles. It is found that temperature shows an increasing behavior as $R$ increases. Physically, a similar effect was also expected because the 
radiation parameter $R$ signifies the relative contribution of conduction heat transfer to thermal radiation transfer. The temperature profiles for different values of the Newtonian heating parameter $\gamma$ are plotted in Figure 12. We observe that temperature profiles increase when $\gamma$ increases. It is due to the fact that, as we increase $\gamma$, it results in an increase in the heat transfer rate from the surface which raises the temperature.

The numerical results for skin friction and Nusselt are shown in Tables 1 and 2. Table 1 is prepared to show the effects of $\operatorname{Pr}, M, K, \mathrm{Gr}, R, \gamma, \beta_{0}, \beta_{1}$, and $\tau$ on the skin frictions $\tau_{0}$ and $\tau_{1}$ corresponding to impulsive and uniformly accelerated motions of the plate, respectively. It is observed that both $\tau_{0}$ and $\tau_{1}$ increase when the values of $\operatorname{Pr}, M, \tau$, and $\beta$ are increased. However, this behavior is quite opposite for radiation parameter $R$, conjugate parameter $\gamma$, permeability parameter $K$, and Grashof number Gr. Table 2 represents the numerical results for the rate of heat transfer in terms of Nusselt number $\mathrm{Nu}$ for different values of Prandtl number Pr, radiation parameter $R$, and conjugate parameter $\gamma$. It is found that the rate of heat transfer decreases with the increasing values of $\operatorname{Pr}$ and increases when $R, \gamma$, and $\tau$ are increased.

\section{Conclusions}

The exact solutions for the unsteady free MHD convection flow of an incompressible Brinkman fluid past a vertical plate in a porous medium with radiation effects and Newtonian heating condition are obtained using the Laplace transform technique. For a detailed discussion of the problem, four important flow situations are discussed. It was observed from the analytical and graphical results that the obtained solutions satisfy the imposed initial and boundary conditions as well as the basic equations of the present problem. The analytical results of velocity corresponding to the impulsive and uniformly accelerated motions of the plate together with temperature are plotted graphically, whereas the numerical results for skin friction and Nusselt number are provided in tables. From the present analysis, we observed the following:

(i) The effects of $M$ and $K$ on the velocities are quite opposite.

(ii) The magnitude of velocity for uniformly accelerated motion is greater than the magnitude for the impulsive motion of the plate.

(iii) In the absence of free convection velocity shows the highest amplitude.

(iv) Increasing thermal radiation increases velocity.

(v) Velocities increase for large values of the Newtonian heating parameter.

(vi) Velocities are increasing functions of time.

(vii) Increasing non-Newtonian fluid parameter $\beta$ decreases fluid velocity.

(viii) The temperature increases for large values of $R$ and $\gamma$.

(ix) The solutions in $[40,41]$ appear as the limiting cases of the present solutions.

\section{Acknowledgment}

The authors would like to acknowledge the Research Management Centre, UTM, for the financial support through vote nos. $4 \mathrm{~F} 109$ and $04 \mathrm{~h} 27$ for this research.

\section{References}

[1] P. Chandran, N. C. Sacheti, and A. K. Singh, "Unsteady hydromagnetic free convection flow with heat flux and accelerated boundary motion," Journal of the Physical Society of Japan, vol. 67, no. 1, pp. 124-129, 1998.

[2] P. Chandran, N. C. Sacheti, and A. K. Singh, "Natural convection near a vertical plate with ramped wall temperature," Heat and Mass Transfer, vol. 41, no. 5, pp. 459-464, 2005.

[3] R. Muthucumaraswamy, "Natural convection on flow past an impulsively started vertical plate with variable surface heat flux," Far East Journal of Applied Mathematics, vol. 14, no. 1, pp. 99119, 2004.

[4] R. Muthucumaraswamy, P. Chandrakala, and S. A. Raj, "Radiative heat and mass transfer effects on moving isothermal vertical plate in the presence of chemical reaction," International Journal of Applied Mechanics and Engineering, vol. 11, no. 3, pp. 639-646, 2006.

[5] R. Muthucumaraswamy and A. Vijayalakshmi, "Effects of heat and mass transfer on flow past an oscillating vertical plate with variable temperature," International Journal of Applied Mathematics and Mechanics, vol. 4, no. 1, pp. 59-65, 2008.

[6] R. Muthucumaraswamy, "Chemical reaction effects on vertical oscillating plate with variable temperature," Chemical Industry and Chemical Engineering Quarterly, vol. 16, no. 2, pp. 167-173, 2010.

[7] G. S. Seth, M. S. Ansari, and R. Nandkeolyar, "MHD natural convection flow with radiative heat transfer past an impulsively moving plate with ramped wall temperature," Heat and Mass Transfer, vol. 47, no. 5, pp. 551-561, 2011.

[8] P. Chandrakala and P. N. Bhaskar, "Thermal radiation effects on MHD flow past a vertical oscillating plate," International Journal of Applied Mechanics and Engineering, vol. 14, no. 2, pp. 349-358, 2009.

[9] P. Chandrakala, "Radiation effects on flow past an impulsively started vertical oscillating plate with uniform heat flux," International Journal of Dynamics of Fluids, vol. 6, pp. 209-215, 2010.

[10] P. Chandrakala and P. N. Bhaskar, "Radiation effects on oscillating vertical plate with uniform heat flux and mass diffusion," International Journal of Fluids Engineering, vol. 4, pp. 1-11, 2012.

[11] J. H. Merkin, "Natural-convection boundary-layer flow on a vertical surface with newtonian heating," International Journal of Heat and Fluid Flow, vol. 15, no. 5, pp. 392-398, 1994.

[12] M. Z. Salleh, R. Nazar, and I. Pop, "Numerical solutions of free convection boundary layer flow on a solid sphere with newtonian heating in a micropolar fluid," Meccanica, vol. 47, no. 5, pp. 1261-1269, 2012.

[13] R. C. Chaudhary and P. Jain, "Unsteady free convection boundary-layer flow past an impulsively started vertical surface with newtonian heating," Romanian Journal of Physics, vol. 51, pp. 911-925, 2006.

[14] R. C. Chaudhary and P. Jain, "An exact solution to the unsteady free-convection boundary-layer flow past an impulsively started vertical surface with newtonian heating," Journal of Engineering Physics and Thermophysics, vol. 80, no. 5, pp. 954-960, 2007. 
[15] S. K. Guchhait, S. Das, and R. N. Jana, "Unsteady free convective flow past a moving vertical porous plate with newtonian heating," International Journal of Engineering Science and Technology, vol. 4, no. 7, pp. 3236-3245, 2012.

[16] P. Mebine and E. M. Adigio, "Unsteady free convection flow with thermal radiation past a vertical porous plate with newtonian heating," Turkish Journal of Physics, vol. 33, no. 2, pp. 109119, 2009.

[17] M. Z. Salleh, R. Nazar, and I. Pop, "Boundary layer flow and heat transfer over a stretching sheet with newtonian heating," Journal of the Taiwan Institute of Chemical Engineers, vol. 41, no. 6, pp. 651-655, 2010.

[18] M. Z. Salleh, R. Nazar, N. M. Arifin, I. Pop, and J. H. Merkin, "Forced-convection heat transfer over a circular cylinder with newtonian heating," Journal of Engineering Mathematics, vol. 69, no. 1, pp. 101-110, 2011.

[19] M. Narahari and A. Ishak, "Radiation effects on free convection flow near a moving vertical plate with newtonian heating," Journal of Applied Sciences, vol. 11, no. 7, pp. 1096-1104, 2011.

[20] N. S. Akbar and S. Nadeem, "Mixed convective magnetohydrodynamic peristaltic flow of a Jeffrey nanofluid with newtonian heating," Zeitschrift für Naturforschung, vol. 68, pp. 433-441, 2013.

[21] M. Narahari and M. N. Yunus, "Free convection flow past an impulsively started infinite vertical plate with newtonian heating in the presence of thermal radiation and mass diffusion," Turkish Journal of Engineering and Environmental Sciences, vol. 35, no. 3, pp. 187-198, 2011.

[22] T. Hayat, M. Awais, and A. Alsaedi, "Newtonian heating and magnetohydrodynamic effects in flow of a Jeffery fluid over a radially stretching surface," International Journal of Physical Sciences, vol. 7, no. 21, pp. 2838-2844, 2012.

[23] D. Lesnic, D. B. Ingham, and I. Pop, "Free convection boundarylayer flow along a vertical surface in a porous medium with newtonian heating," International Journal of Heat and Mass Transfer, vol. 42, no. 14, pp. 2621-2627, 1999.

[24] D. Lesnic, D. B. Ingham, and I. Pop, "Free convection from a horizontal surface in a porous medium with newtonian heating," Journal of Porous Media, vol. 3, no. 3, pp. 227-235, 2000.

[25] D. Lesnic, D. B. Ingham, I. Pop, and C. Storr, "Free convection boundary-layer flow above a nearly horizontal surface in a porous medium with newtonian heating," Heat and Mass Transfer, vol. 40, no. 9, pp. 665-672, 2004.

[26] I. Khan, K. Fakhar, and S. Shafie, "Magnetohydrodynamic free convection flow past an oscillating plate embedded in a porous medium," Journal of the Physical Society of Japan, vol. 80, no. 10, Article ID 104401, 2011.

[27] S. U. Haq, C. Fetecau, I. Khan, F. Ali, and S. Shafie, "Radiation and porosity effects on the magnetohydrodynamic flow past an oscillating vertical plate with uniform heat flux," Zeitschrift für Naturforschung A, vol. 67a, pp. 572-580, 2012.

[28] A. H. Mohammed, I. Khan, and S. Shafie, "Exact solutions of MHD natural convection transient flow near an oscillating plate emerged in a porous medium," Jurnal Teknologi, vol. 57, no. 1, pp. 1-16, 2012.

[29] W. A. Khan and I. Pop, "Boundary-layer flow of a nanofluid past a stretching sheet," International Journal of Heat and Mass Transfer, vol. 53, no. 11-12, pp. 2477-2483, 2010.

[30] W. A. Khan and A. Aziz, "Natural convection flow of a nanofluid over a vertical plate with uniform surface heat flux,"
International Journal of Thermal Sciences, vol. 50, no. 7, pp. 12071214, 2011.

[31] O. D. Makinde, W. A. Khan, and Z. H. Khan, "Buoyancy effects on MHD stagnation point flow and heat transfer of a nanofluid past a convectively heated stretching/shrinking sheet," International Journal of Heat and Mass Transfer, vol. 62, pp. 526-533, 2013.

[32] N. S. Akbar and S. Nadeem, "Simulation of heat transfer on the peristaltic flow of a Jeffrey-six constant fluid in a diverging tube," International Communications in Heat and Mass Transfer, vol. 38, no. 2, pp. 154-159, 2011.

[33] N. S. Akbar and S. Nadeem, "Characteristics of heating scheme and mass transfer on the peristaltic flow for an Eyring-Powell fluid in an endoscope," International Journal of Heat and Mass Transfer, vol. 55, no. 1-3, pp. 375-383, 2012.

[34] H. C. Brinkman, "A calculation of the viscous force exerted by a flowing fluid on a dense swarm of particles," Applied Scientific Research, vol. 1, no. 1, pp. 27-34, 1949.

[35] H. C. Brinkman, "On the permeability of media consisting of closely packed porous particles," Applied Scientific Research, vol. 1, no. 1, pp. 81-86, 1949.

[36] C. T. Hsu and P. Cheng, "The Brinkman model for natural convection about a semi-infinite vertical flat plate in a porous medium," International Journal of Heat and Mass Transfer, vol. 28, no. 3, pp. 683-697, 1985.

[37] S. V. Varma and M. S. Babu, "Brinkman model for MHD viscous incompressible flow through a porous channel," Indian Journal of Pure and Applied Mathematics, vol. 16, no. 7, pp. 796-806, 1985.

[38] R. S. R. Gorla, M. A. Mansour, and M. Gaid Sahar, "Natural convection from a vertical plate in a porous medium using Brinkman's model," Transport in Porous Media, vol. 36, no. 3, pp. 357-371, 1999.

[39] K. R. Rajagopal, "On a hierarchy of approximate models for flows of incompressible fluids through porous solids," Mathematical Models and Methods in Applied Sciences, vol. 17, no. 2, article 215, 2007.

[40] C. Fetecǎu, C. Fetecǎu, and M. A. Imran, "On stokes problems for fluids of Brinkman type," Mathematical Reports, vol. 13, no. 1, pp. 15-26, 2011.

[41] F. Ali, I. Khan, Samiulhaq, and S. Shafie, "A note on new exact solutions for some unsteady flows of Brinkman-type fluids over a plane wall," Zeitschrift für Naturforschung A, vol. 67, pp. 377380, 2012.

[42] K. R. Cramer and S. I. Pai, Magnetofluid Dynamics For Engineers and Applied PhysiCists, McGraw-Hill, New York, NY, USA, 1973. 


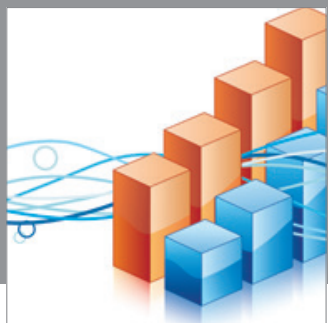

Advances in

Operations Research

mansans

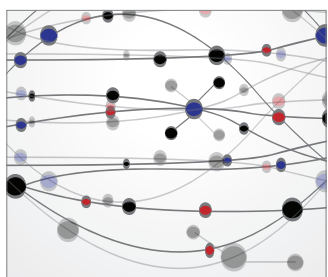

The Scientific World Journal
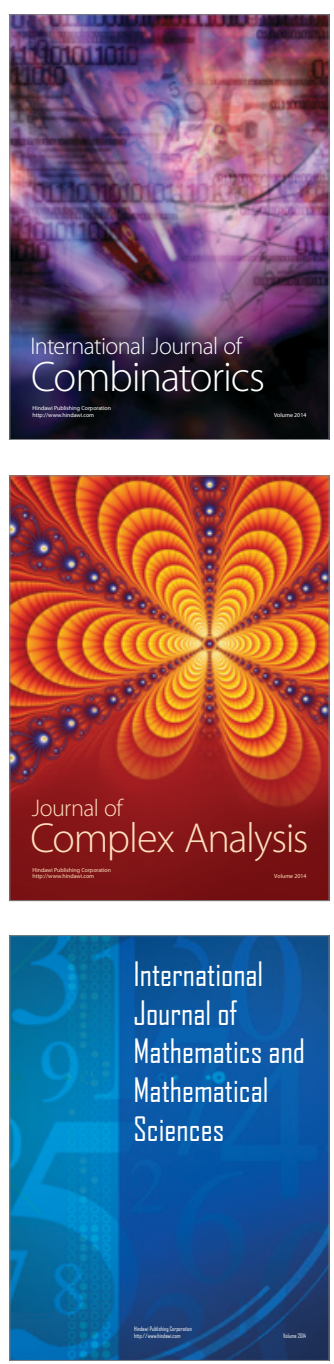
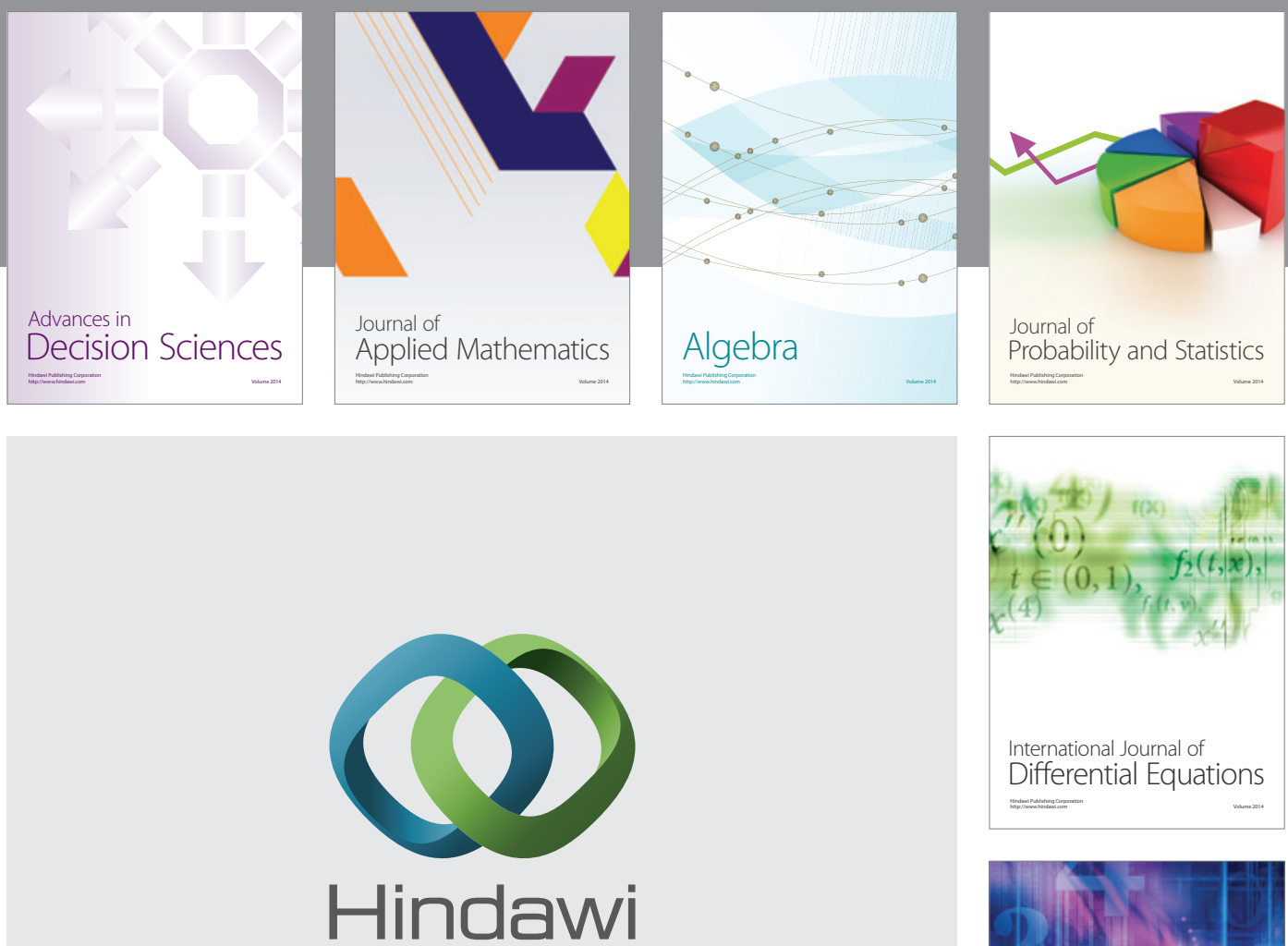

Submit your manuscripts at http://www.hindawi.com
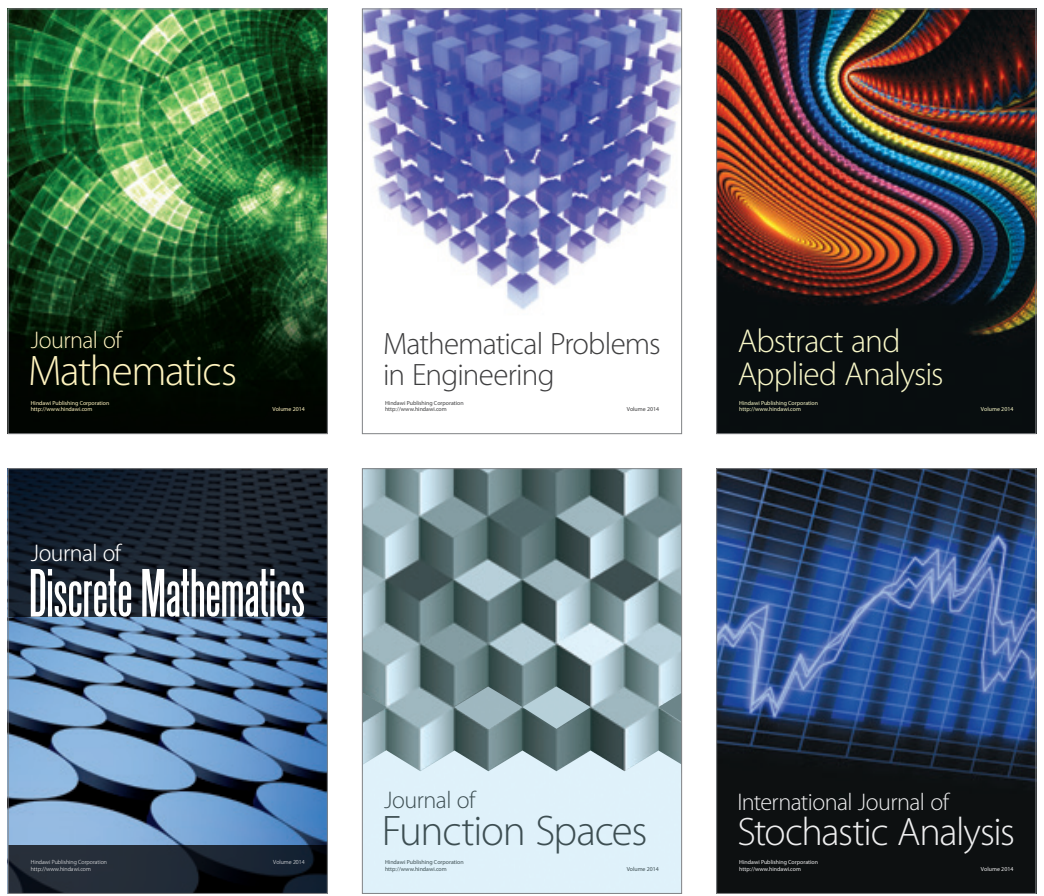

Journal of

Function Spaces

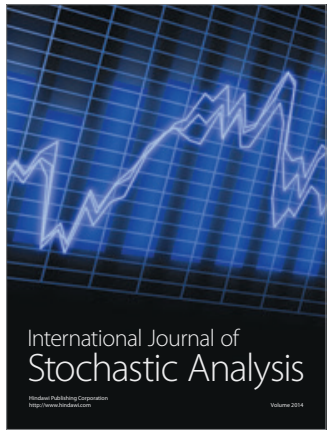

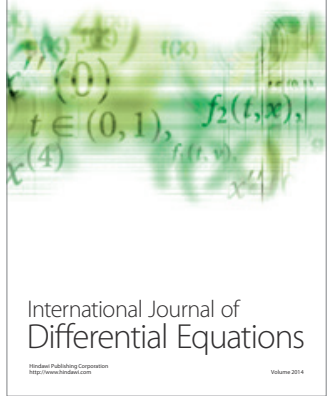
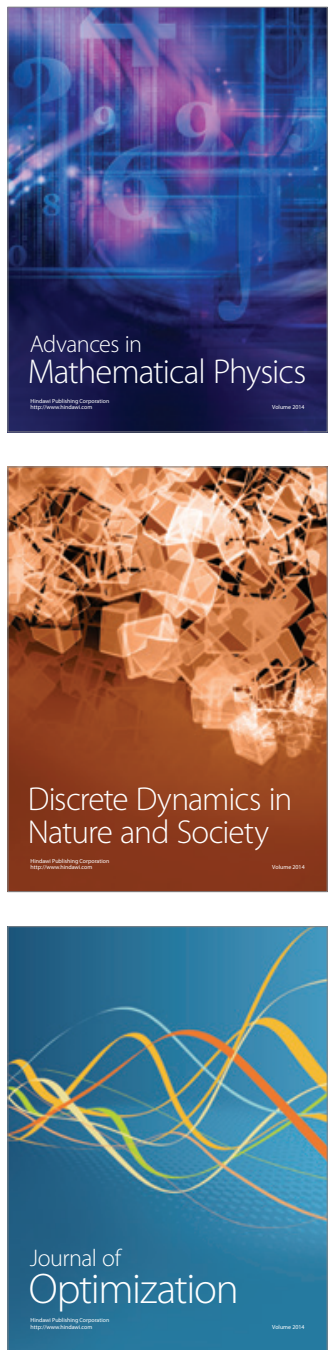